\title{
Perturbative approach to higher derivative theories with fermions
}

\author{
Tai-Chung Cheng, Pei-Ming Ho, ${ }^{*}$ and Mao-Chuang Yeh \\ Department of Physics, National Taiwan University, Taipei 106, Taiwan, Republic of China
}

(Received 4 July 2002; published 21 October 2002)

\begin{abstract}
We extend the perturbative approach developed in an earlier work to deal with Lagrangians which have arbitrary higher order time derivative terms for both bosons and fermions. This approach enables us to find an effective Lagrangian with only first time derivatives order by order in the coupling constant. As in the pure bosonic case, to the first order, the quantized Hamiltonian is bounded from below whenever the potential is. We show in the example of a single complex fermion that higher derivative interactions result in an effective mass and change of vacuum for the low energy modes. The supersymmetric noncommutative Wess-Zumino model is considered as another example. We also comment on the higher derivative terms in Witten's string field theory and the effectiveness of level truncation.
\end{abstract}

DOI: 10.1103/PhysRevD.66.085015

PACS number(s): 11.10.Ef, 03.70.+k

\section{INTRODUCTION}

It is generally assumed that the equation of motion for a bosonic variable is a second order differential equation, and that for a fermionic variable is first order. Problems arise immediately when modifications by higher derivative terms are introduced. For example, even in the classical regime, acausal behavior or runaway solutions appear for charged point particles due to the radiation reaction which is a third derivative term. In general, the canonical formulation [1] always leads to a spectrum which is unbounded from below for higher derivative theories. For quantum field theories, higher derivatives often imply nonrenormalizability or violation of unitarity. However, there are counterexamples [2], and in fact higher derivatives can be used to improve the behavior of quantum field theories by regularizing the ultraviolet divergences [3]. Moreover, apart from the technical difficulty, there is no known physical reason why nature should abhor higher derivative interactions. We are justified to ignore higher derivatives only in the low energy limit, and it would be a great puzzle if we never need higher derivative terms in formulating fundamental theories. In fact, in string field theory there are indeed infinite higher derivatives. For instance, in Witten's bosonic open string field theory [4], any field $f$ appears in the interaction as [5]

$$
\widetilde{f} \equiv e^{a^{2} \partial_{\mu} \partial^{\mu}} f
$$

where $a^{2}=\ln (3 \sqrt{3} / 4) \alpha^{\prime}$. See [6] for references to higher derivative (and nonlocal) theories and their applications.

In a previous work [6], we considered a perturbative approach to higher derivative theories, which is equivalent to the approach of [7]. It deals with Lagrangians whose kinetic term is the same as that for ordinary free fields, and all higher derivative terms appear only in the interaction terms. In a perturbative expansion of the coupling constant, higher derivative terms are replaced by lower derivative terms. An effective Lagrangian is obtained in the end with only first derivatives, and its quantization is straightforward. This for-

*Email address: pmho@phys.ntu.edu.tw mulation is closely related to the approach of Yang and Feldman [8] which directly deals with the equations of motion at the quantum level. Recently, it was shown that the perturbative treatment of field theories on noncommutative spacetime in the spirit of $[8]$ is unitary $[9,10]$.

The physical motivation for the perturbative approach is the following. To extend the range of validity of a physical model to higher energies, we might need to add new interaction terms with higher derivatives. However, no matter how small the coupling is, this would imply a sudden increase in the dimension of phase space according to the canonical formulation. Furthermore, the Hamiltonian always becomes unbounded from below. It is thus natural to carry out a projection back to the original phase space, which is called the "reduced phase space," so that the new interaction term will not abruptly change the theory into a completely new theory that we do not know how to handle. We showed that the Hamiltonian on the reduced phase space is bounded from below at least to the first order [6] (whenever the potential is).

To demonstrate the physical meaning of the perturbative approach more explicitly, we showed in the example of a coupled spring system [6] that the perturbative approach gives the correct description of the normal mode with lower natural frequency while ignoring the other normal mode with higher natural frequency.

The purpose of this paper is to extend our previous work to include fermions, and to examine the effects of higher derivative interactions on fermionic fields. Interesting results are obtained. Because of the anticommutativity of fermions, the effect of higher derivative terms is more severely restricted than bosons in the perturbative approach.

For the case of a single complex fermion in $0+1$ dimension, we find that, to all orders, the effect of arbitrary higher derivative interactions is always equivalent to a change of vacuum and an effective mass.

A natural question about higher derivative theories is whether supersymmetry can cure the problem of the Hamiltonian being unbounded from below. We will show in an example that in the canonical formulation, the quantization of fermions with higher derivatives will destroy the reality conditions. Thus the supercharge $Q$ will not be Hermitian, 
and we can not conclude that $H \geqslant 0$ from the supersymmetric (SUSY) algebra $H \sim Q^{2}$. We will apply the perturbative approach to the 1+1-dimensional supersymmetric noncommutative Wess-Zumino model [11] as an example. The perturbative approach not only provides a consistent quantization but also preserves supersymmetry.

Another interesting observation we make in this paper is that the higher derivatives in Eq. (1) effectively increase the interaction strength for fields of higher levels. This makes the technique of level truncation less effective for fluctuation modes compared with the zero modes in the string field theory.

This paper is organized as follows. We show in Sec. II that higher derivative terms introduce a different problem for fermions in the canonical formulation. The problem is that canonical quantization is not consistent with the reality (Hermiticity) condition on fields. In Sec. III, we extend our previous work to the generic case with arbitrary numbers of bosons and fermions. Explicit results to the first order are given, showing that the Hamiltonian becomes bounded from below when the potential is. We describe how to carry out this procedure to an arbitrary order. We prove that the perturbative approach is consistent to all orders and that the effect of higher derivative terms is properly preserved in this approach. The case of a single complex fermion in $0+1$ dimension is considered as a simple example. We find that the effect of any higher derivative interactions for this case sums up to a change of vacuum and an effective mass, to arbitrary orders. We also consider the noncommutative WessZumino model to the first order as another example. For Witten's open string field theory, we find that the higher derivatives in Eq. (1) make level truncation less effective for fluctuation modes. These examples are given in Sec. IV. Finally, in Sec. V, we further extend our approach to a larger reduced phase space which contains fields with derivatives up to any finite order.

\section{CANONICAL FORMULATION}

Consider the canonical formulation with higher time derivatives for both bosons and fermions. The Lagrangian is

$$
L_{0}\left(\Phi_{a}^{(i)}, \Psi_{a^{\prime}}^{(j)}\right)
$$

where $\Phi_{a}^{(i)}\left(\Psi_{a^{\prime}}^{(j)}\right)$ is the $i(j)$ th time derivative of the $a\left(a^{\prime}\right)$ th boson (fermion), $a\left(a^{\prime}\right)=1 \cdots M_{B}\left(M_{F}\right)$ and $i(j)$ $=1 \cdots N_{B}\left(N_{F}\right)$.

Let us apply the formalism of [12]. The variation of the action

$$
S=\int_{t_{i}}^{t_{f}} d t L_{0}
$$

with respect to $\Phi_{a}, \Psi_{a^{\prime}}$ is found to be the time integral of the Euler-Lagrange equations

$$
\sum_{i=0}^{N_{B}}\left(-\frac{d}{d t}\right)^{i} \frac{\partial L_{0}}{\partial \Phi_{a}^{(i)}}=0,
$$

$$
\sum_{j=0}^{N_{F}}\left(-\frac{d}{d t}\right)^{j} \frac{\partial L_{0}}{\partial \Psi_{a^{\prime}}^{(j)}}=0
$$

multiplied by $\delta \Phi_{a}, \delta \Psi_{a^{\prime}}$ from the right, plus a boundary term

$$
\left[\sum_{a=1}^{M_{B}} \sum_{i=0}^{N_{B}-1} P_{a i} \delta \Phi_{a}^{(i)}+\sum_{a^{\prime}=1}^{M_{F}} \sum_{j=0}^{N_{F}-1} Q_{a^{\prime} j} \delta \Psi_{a^{\prime}}^{(j)}\right]_{t_{i}}^{t_{f}},
$$

where $P_{a i}$ and $Q_{a^{\prime} j}$ are the conjugate momenta of $\Phi_{a}^{(i)}$ and $\Psi_{a^{\prime}}^{(j)}$ given by

$$
\begin{aligned}
P_{a i} & =\sum_{k=0}^{N_{B}-i-1}\left(-\frac{d}{d t}\right)^{k} \frac{\partial L_{0}}{\partial \Phi_{a}^{(i+k+1)}}, \\
Q_{a^{\prime} j} & =\sum_{h=0}^{N_{F}-j-1}\left(-\frac{d}{d t}\right)^{h} \frac{\partial L_{0}}{\partial \Psi_{a^{\prime}}^{(j+h+1)}} .
\end{aligned}
$$

In the above, $\partial / \partial \Psi$ is defined to be the derivative with respect to $\Psi$ from the right. The symplectic structure can be directly read off from the boundary term (5) as

$$
\Omega=\sum_{a=1}^{M_{B}} \sum_{i=0}^{N_{B}-1} d P_{a i} d \Phi_{a}^{(i)}+\sum_{a^{\prime}=1}^{M_{F}} \sum_{j=0}^{N_{F}-1} d Q_{a^{\prime} j} d \Psi_{a^{\prime}}^{(j)}
$$

Note that $d \Phi$ 's are anticommuting variables while $d \Psi$ 's are commuting variables.

In the canonical formulation, the Hamiltonian is

$$
H=\sum_{a=1}^{M_{B}} \sum_{i=0}^{N_{B}-1} P_{a i} \Phi_{a}^{(i)}+\sum_{a^{\prime}=1}^{M_{F}} \sum_{j=0}^{N_{F}-1} Q_{a^{\prime} j} \Psi_{a^{\prime}}^{(j)}-L_{0},
$$

assuming that the action is nondegenerate, that is, the definition of $P_{a\left(N_{B}-1\right)}$ in Eq. (6) can be used to solve $\Phi_{a}^{N_{B}}$ as a function of $P_{a\left(N_{B}-1\right)}$ and $\Phi$ 's. This implies that the Hamiltonian is unbounded from below because it is linear in all $P_{a i}$ for $i<\left(N_{B}-1\right)$. Thus we expect violation of unitarity after standard quantization of the system.

Now we consider the fermions. The classical Hamiltonian of a fermion is typically unbounded from below even without higher derivatives. Yet canonical quantization leads to a spectrum which is bounded from below by filling the Dirac sea. This difference between the bosons and fermions raises a question. What happens if we have a higher derivative theory with supersymmetry, where the bosonic spectrum should be identical to the fermionic spectrum?

It turns out that the problem of femions with higher time derivatives is that the canonical quantization is inconsistent with the reality conditions, and thus violation of unitarity is also expected. Let us consider the simplest example of a real fermion $\psi$ in $0+1$ dimension with the Lagrangian

$$
L_{0}=i \psi \dot{\psi}+i g \dot{\psi} \ddot{\psi}
$$

The symplectic form is 


$$
\Omega=i\left(d \phi d \phi+g d \dot{\psi} d \dot{\psi}-g^{2} d \ddot{\psi} d \ddot{\psi}\right),
$$

where $\phi \equiv \psi-g \ddot{\psi}$. The Poisson brackets are

$$
(\phi, \phi)=i, \quad(\dot{\psi}, \dot{\psi})=i / g, \quad(\ddot{\psi}, \ddot{\psi})=-i / g^{2} .
$$

Upon quantization, we replace Poisson brackets by anticommutators for fermions, up to a factor of $\pm i$. For the two possible choices of sign,

$$
\{\cdot, \cdot\}=i(\cdot, \cdot) \text { or }\{\cdot, \cdot\}=-i(\cdot, \cdot) \text {, }
$$

we will have either

$$
\phi^{2}<0 \quad \text { or } \quad \ddot{\psi}^{2}<0 \text {, }
$$

which is inconsistent with the fact that $\psi$ is real. This implies that the supercharge $Q$ is not Hermitian. In fact the quantization is simply inconsistent. Hence we find that the problem for fermions with higher derivative Lagrangians can be even more serious than bosons.

\section{PERTURBATIVE APPROACH}

In this paper we focus on Lagrangians of the form

$$
L=L_{B}+L_{F}-\lambda V
$$

where $\lambda$ is the coupling constant, $L_{B}$ and $L_{F}$ are the free field Lagrangians for bosons and fermions, and $V$ is the interaction piece where higher derivative terms reside. More explicitly, we have

$$
\begin{aligned}
L_{B} & =\sum_{a} \frac{1}{2} \dot{\Phi}_{a}^{2}-\sum_{a} \frac{1}{2} m_{a b}^{B} \Phi_{a} \Phi_{b}, \\
L_{F} & =\sum_{a^{\prime}} i \frac{1}{2} \Psi_{a^{\prime}} \dot{\Psi}_{a^{\prime}}-\sum_{a^{\prime}, b^{\prime}} i \frac{1}{2} m_{a^{\prime} b^{\prime}}^{F} \Psi_{a^{\prime}} \Psi_{b^{\prime}}, \\
V & =V\left(\Phi_{a}^{(i)}, \Psi_{a^{\prime}}^{(j)}\right),
\end{aligned}
$$

where $m^{B}$ is symmetric and $m^{F}$ is antisymmetric. We will give the prescription for the perturbative approach for such Lagrangians in this section.

To be general, let us consider the cases with infinite order time derivatives. Under variation the action is

$$
\begin{aligned}
\delta S= & -\int d t\left(\sum_{a}\left(\mathrm{EOM}_{B}\right)_{a} \delta \Phi_{a}+\sum_{b^{\prime}}\left(\mathrm{EOM}_{F}\right)_{b^{\prime}} \delta \Psi_{b^{\prime}}\right) \\
& +\left[\sum_{k=0}^{\infty} P_{a k} \delta \Phi_{a}^{(k)}+\sum_{h=0}^{\infty} Q_{b^{\prime} h} \delta \Psi_{b^{\prime}}^{(h)}\right]_{t_{i}}^{t_{f}}
\end{aligned}
$$

where the equations of motion for bosons and fermions are

$$
\begin{aligned}
\left(\mathrm{EOM}_{B}\right)_{a} \equiv & \Phi_{a}+\sum_{b} m_{a b}^{B} \Phi_{b} \\
& +\lambda \sum_{i=0}^{\infty}\left(-\frac{d}{d t}\right)^{i} \frac{\partial V}{\partial \Phi_{a}^{(i)}}, \\
\left(\mathrm{EOM}_{F}\right)_{a^{\prime}} \equiv & i \Psi_{a^{\prime}}-i \sum_{b^{\prime}} m_{a^{\prime} b^{\prime}}^{F} \Psi_{b^{\prime}} \\
& +\lambda \sum_{i=0}^{\infty}\left(-\frac{d}{d t}\right)^{i} \frac{\partial V}{\partial \Psi_{a^{\prime}}^{(i)}}
\end{aligned}
$$

According to Eqs. (6) and (7), the canonical momenta $P_{a}$ and $Q_{b^{\prime}}$ are

$$
\begin{gathered}
P_{a k}=\dot{\Phi}_{a} \delta_{k 0}-\lambda \sum_{i=k+1}^{\infty}\left(-\frac{d}{d t}\right)^{(i-k-1)} \frac{\partial V}{\partial \Phi_{a}^{(i)}}, \\
Q_{a^{\prime} k}=\frac{1}{2} \Psi_{a^{\prime}} \delta_{k 0}-\lambda \sum_{i=k+1}^{\infty}\left(-\frac{d}{d t}\right)^{(i-k-1)} \frac{\partial V}{\partial \Psi_{a^{\prime}}^{(i)}} .
\end{gathered}
$$

The symplectic two-form is given by Eq. (8).

\section{A. First order approximation}

Following [6], we will construct the effective action without higher derivatives for a reduced phase space which is appropriate for the low energy, weak coupling regime. We will keep $\Psi$ for fermions and $\Phi, \Phi$ for bosons as the variables for our reduced phase space. Our strategy is to use the equations of motion to replace higher derivative terms by lower derivative ones. To the lowest order,

$$
\begin{aligned}
& \Phi_{a}^{(n)} \simeq \begin{cases}\sum_{b} M_{a b}^{B(n / 2)} \Phi_{b} & (n=\text { even }), \\
\sum_{b} M_{a b}^{B[(n-1) / 2]} \dot{\Phi}_{b} & (n=\text { odd }),\end{cases} \\
& \Psi_{a^{\prime}}^{(n)} \simeq \sum_{b^{\prime}} M_{a^{\prime} b^{\prime}}^{F(n)} \Psi_{b^{\prime}},
\end{aligned}
$$

where

$$
M_{a_{n} a_{0}}^{B(n)} \equiv\left\{\begin{array}{l}
\sum_{a_{n-1}} \cdots \sum_{a_{1}}\left(-m_{a_{n} a_{n-1}}^{B}\right) \cdots\left(-m_{a_{1} a_{0}}^{B}\right) \quad(n \geqslant 2), \\
-m_{a_{1} a_{0}}^{B} \quad(n=1), \\
\delta_{a_{n} a_{0}} \quad(n=0)
\end{array}\right.
$$




$$
M_{a_{n}^{\prime} a_{0}^{\prime}}^{F(n)} \equiv \begin{cases}\sum_{a_{n-1}^{\prime}} \cdots \sum_{a_{1}^{\prime}}\left(m_{a_{n}^{\prime} a_{n-1}^{\prime}}^{F}\right) \cdots\left(m_{a_{1}^{\prime} a_{0}^{\prime}}^{F} \quad(n \geqslant 2),\right. \\ m_{a_{1}^{\prime} a_{0}^{\prime}} \quad(n=1), \\ \delta_{a_{n}^{\prime} a_{0}^{\prime}}(n=0) .\end{cases}
$$

The symplectic two-form (8) reduces to

$$
[\Omega]_{1}=\sum_{a} d p_{a 0} d \Phi_{a}+d p_{a 1} d \Phi_{a}+\sum_{a^{\prime}} d q_{a^{\prime}} d \Psi_{a^{\prime}},
$$

where

$$
\begin{aligned}
& p_{a 0}=\dot{\Phi}_{a}-\lambda \xi_{a 0}, \quad p_{a 1}=-\lambda \xi_{a 1}, \\
& q_{a^{\prime}}=\frac{i}{2} \Psi_{a^{\prime}}-\lambda \xi_{a^{\prime} 2},
\end{aligned}
$$

and

$$
\begin{gathered}
\xi_{a 0}=\sum_{b=1}^{M_{B}} \sum_{j=0}^{\infty} \sum_{i=2 j+1}^{\infty} M_{a b}^{B(j)}\left[\left(-\frac{d}{d t}\right)^{i-2 j-1} \frac{\partial V}{\partial \Phi_{b}^{(i)}}\right]_{1}, \\
\xi_{a 1}=\sum_{b=1}^{M_{B}} \sum_{j=0}^{\infty} \sum_{i=2 j+2}^{\infty} M_{a b}^{B(j)}\left[\left(-\frac{d}{d t}\right)^{i-2 j-2} \frac{\partial V}{\partial \Phi_{b}^{(i)}}\right]_{1}, \\
\xi_{a^{\prime} 2}=\sum_{b^{\prime}=1}^{M_{F}} \sum_{j=0}^{\infty} \sum_{i=j+1}^{\infty} M_{b^{\prime} a^{\prime}}^{F(j)}\left[\left(-\frac{d}{d t}\right)^{i-j-1} \frac{\partial V}{\partial \Psi_{b^{\prime}}^{(i)}}\right]_{1} .
\end{gathered}
$$

Here $[\cdot]_{1}$ refers to the replacement of higher derivative terms by functions of $\Phi, \Phi$, and $\Psi$ via Eq. (24).

Explicitly, the symplectic two-form is

$$
\begin{aligned}
{[\Omega]_{1}=} & \sum_{a b}\left\{\left[-\delta_{a b}+\lambda\left(\frac{\partial \xi_{a 0}}{\partial \dot{\Phi}_{b}}-\frac{\partial \xi_{b 1}}{\partial \Phi_{a}}\right)\right] d \Phi_{a} d \dot{\Phi}_{b}\right. \\
& \left.+\lambda \frac{\partial \xi_{a 0}}{\partial \Phi_{b}} d \Phi_{a} d \Phi_{b}+\lambda \frac{\partial \xi_{a 1}}{\partial \Phi_{b}} d \dot{\Phi}_{a} d \dot{\Phi}_{b}\right\} \\
& +\sum_{a^{\prime} b^{\prime}}\left[i \frac{\delta_{a^{\prime} b^{\prime}}}{2}-\lambda \frac{\partial_{R} \xi_{a^{\prime} 2}}{\partial \Psi_{b^{\prime}}}\right] d \Psi_{a^{\prime}} d \Psi_{b^{\prime}} \\
& +\sum_{a, a^{\prime}}\left\{\lambda\left(\frac{\partial_{R} \xi_{a 0}}{\partial \Psi_{a^{\prime}}}-\frac{\partial \xi_{a^{\prime} 2}}{\partial \Phi_{a}}\right) d \Phi_{a} d \Psi_{a^{\prime}}\right. \\
& \left.+\lambda\left(\frac{\partial_{R} \xi_{a 1}}{\partial \Psi_{a^{\prime}}}-\frac{\partial \xi_{a^{\prime} 2}}{\partial \dot{\Phi}_{a}}\right) d \Phi_{a} d \Psi_{a^{\prime}}\right\} .
\end{aligned}
$$

Inverting the symplectic two-form, we find the Poisson brackets to the lowest order in $\lambda$

$$
\begin{gathered}
\left(\Phi_{a}, \dot{\Phi}_{b}\right)=\delta_{a b}+\lambda\left(\frac{\partial \xi_{b 0}}{\partial \Phi_{a}}-\frac{\partial \xi_{a 1}}{\partial \Phi_{b}}\right) \\
\left(\Phi_{a}, \Phi_{b}\right)=\lambda\left(\frac{\partial \xi_{a 1}}{\partial \dot{\Phi}_{b}}-\frac{\partial \xi_{b 1}}{\partial \Phi_{a}}\right), \\
\left(\Phi_{a}, \Phi_{b}\right)=\lambda\left(\frac{\partial \xi_{a 0}}{\partial \Phi_{b}}-\frac{\partial \xi_{b 0}}{\partial \Phi_{a}}\right), \\
\left(\Psi_{a^{\prime}}, \Psi_{b^{\prime}}\right)=-i \delta_{a^{\prime} b^{\prime}}-\lambda\left(\frac{\partial \xi_{b^{\prime} 2}}{\partial \Psi_{a^{\prime}}}+\frac{\partial \xi_{a^{\prime} 2}}{\partial \Psi_{b^{\prime}}}\right),
\end{gathered}
$$

$$
\begin{aligned}
& \left(\Phi_{a}, \Psi_{b^{\prime}}\right)=-i \lambda\left(\frac{\partial \xi_{b^{\prime} 2}}{\partial \dot{\Phi}_{a}}-\frac{\partial \xi_{a 1}}{\partial \Psi_{b^{\prime}}}\right), \\
& \left(\dot{\Phi}_{a}, \Psi_{b^{\prime}}\right)=i \lambda\left(\frac{\partial \xi_{b^{\prime} 2}}{\partial \Phi_{a}}-\frac{\partial \xi_{a 0}}{\partial \Psi_{b^{\prime}}}\right) .
\end{aligned}
$$

Remarkably, by a simple change of variables

$$
\begin{aligned}
\varphi_{a} & =\Phi_{a}+\lambda \xi_{a 1}, \quad \pi_{a}=\Phi_{a}-\lambda \xi_{a 0}, \\
\psi_{a^{\prime}} & =\Psi_{a^{\prime}}+i \lambda \xi_{a^{\prime} 2},
\end{aligned}
$$

the Poisson brackets can be put in the standard form

$$
\left(\varphi_{a}, \pi_{b}\right)=\delta_{a b}, \quad\left(\psi_{a^{\prime}}, \psi_{b^{\prime}}\right)=-i \delta_{a^{\prime} b^{\prime}},
$$

with all other Poisson brackets vanishing.

The Hamiltonian for the reduced phase space variables is defined as

$$
\widetilde{H}_{1}=\left[\sum_{a}\left(p_{a 0} \dot{\Phi}_{a}+p_{a 1} \ddot{\Phi}_{a}\right)+\sum_{a^{\prime}} q_{a^{\prime}} \dot{\Psi}_{a^{\prime}}-L\right]_{1} .
$$

In terms of the new variables $\varphi, \pi$, and $\psi$, it is

$$
\begin{aligned}
\widetilde{H}_{1}= & \sum_{a} \frac{1}{2} \pi_{a}^{2}+\sum_{a, b} \frac{1}{2} m_{a b}^{B} \varphi_{a} \varphi_{b}+\sum_{a^{\prime}, b^{\prime}} \frac{i}{2} m_{a^{\prime} b^{\prime}}^{F} \psi_{a^{\prime}} \psi_{b^{\prime}} \\
& +\lambda[V]_{1}(\varphi, \pi, \psi)
\end{aligned}
$$

Note that, if the potential $V$ is bounded from below, the first order Hamiltonian is also bounded from below. One can check that the Hamilton equations

$$
\dot{\varphi}_{a}=\left(\varphi_{a}, \widetilde{H}_{1}\right), \quad \dot{\pi}_{a}=\left(\pi_{a}, \widetilde{H}_{1}\right), \quad \dot{\psi}_{a^{\prime}}=\left(\psi_{a^{\prime}}, \widetilde{H}_{1}\right)
$$

reproduce the equations of motion (20) and (21) to the first order in $\lambda$.

For the reduced phase space, the effective Lagrangian corresponding to the Hamiltonian (43) is found to be 


$$
\begin{aligned}
\tilde{L}_{1}= & \frac{1}{2} \sum_{a} \dot{\varphi}_{a}^{2}-\sum_{a, b} \frac{m_{a b}^{B}}{2} \varphi_{a} \varphi_{b}+\sum_{a^{\prime}} \frac{1}{2} i \psi_{a^{\prime}} \dot{\psi}_{a^{\prime}} \\
& -\sum_{a^{\prime} b^{\prime}} \frac{1}{2} i m_{a^{\prime} b^{\prime}}^{F} \psi_{a^{\prime}} \psi_{b^{\prime}}-\lambda[V]_{1}(\varphi, \dot{\varphi}, \psi) .
\end{aligned}
$$

Its Euler-Lagrange equation agrees with the original system to the first order in $\lambda$. For this construction of the effective Lagrangian to be self-consistent, we also need the conjugate momenta of $\varphi$ and $\psi$ defined from the effective Lagrangian (44) to agree with Eq. (39). While the consistency for the fermions is trivial, for the bosons we need to use the identity

$$
\xi_{a 0}+\left[\xi_{a 1}\right]_{1}=\frac{\partial[V]_{1}}{\partial \dot{\Phi}_{a}},
$$

which can be verified using Eqs. (29) and (30). Since the final expression of the Lagrangian (44) contains only first derivatives, its quantization is straightforward.

The general result Eq. (44), is very useful. It says that, to the first order approximation, in terms of some new variables, the effective Lagrangian is formally the same as simply reducing all higher dervative terms in the original Lagrangian to lower derivatives according to the free field equations.
As Lagrangians are only defined up to total derivatives, let us comment on the difference total derivative terms can make. Starting with two Lagrangians differing from each other only by total derivatives, their effective actions (44) will appear to be different. But this difference simply originates from a different definition of the variables $\varphi, \psi$, and the effective Lagrangians are in fact equivalent.

\section{B. Higher order approximation}

For higher order corrections, we first iterate the equations of motion (20) and (21) up to a certain order $\mathcal{O}\left(g^{n}\right)$. For example, to the first order,

$$
\begin{gathered}
\ddot{\Phi}_{a} \rightarrow-\sum_{b} m_{a b}^{B} \Phi_{b}-\lambda \sum_{i=0}^{\infty}\left[\left(-\frac{d}{d t}\right)^{i} \frac{\partial V}{\partial \Phi_{a}^{(i)}}\right]_{1}, \\
\dot{\Psi}_{a^{\prime}} \rightarrow \sum_{b^{\prime}} m_{a^{\prime} b^{\prime}}^{F} \Psi_{b^{\prime}}+i \lambda \sum_{j=0}^{\infty}\left[\left(-\frac{d}{d t}\right)^{j} \frac{\partial V}{\partial \Psi_{a^{\prime}}^{(j)}}\right]_{1} .
\end{gathered}
$$

Higher derivatives of $\Phi_{a}, \Psi_{a^{\prime}}$ can also be replaced by functions of $\Phi_{a}, \dot{\Phi}_{a}$, and $\Psi_{a^{\prime}}$ up to the same order in $\lambda$ by differentiating with respect to time and repeatedly using Eqs. (46) and (47) as

$$
\begin{aligned}
& \Phi_{a}^{(n)} \simeq\left\{\begin{array}{l}
\sum_{b}\left(M_{a b}^{B(n / 2)} \Phi_{b}-\lambda \sum_{l=1}^{n / 2} \sum_{k=0}^{\infty}\left[M_{a b}^{B(n / 2-l)}\left(-\frac{d}{d t}\right)^{k+2 l-2} \frac{\partial V}{\partial \Phi_{b}^{(k)}}\right]\right)_{1} \quad(n=\mathrm{even}), \\
\sum_{b}\left(M_{a, b}^{B(n-1) / 2} \Phi+\lambda \sum_{l=1}^{(n-1) / 2} \sum_{k=0}^{\infty}\left[M_{a b}^{B[(n-1) / 2-l]}\left(-\frac{d}{d t}\right)^{k+2 l-1} \frac{\partial V}{\partial \Phi_{b}^{(k)}}\right]\right) \quad(n=\text { odd }),
\end{array}\right. \\
& \Psi_{a^{\prime}}^{(n)} \simeq \sum_{b^{\prime}}\left(M_{a^{\prime} b^{\prime}}^{(n)} \Psi_{b^{\prime}}+i \lambda \sum_{j=0}^{\infty} \sum_{l=1}^{n}\left[M_{a^{\prime} b^{\prime}}^{F(n-l)}\left(-\frac{d}{d t}\right)^{j+l-1} \frac{\partial V}{\partial \Psi_{b^{\prime}}^{(j)}}\right]_{1}\right) .
\end{aligned}
$$

In general, we can always have all $\Phi_{a}^{(n)}, \Psi_{a^{\prime}}^{(n)}$ expressed as functions of $\Phi_{a}, \dot{\Phi}_{a}$, and $\Psi_{a^{\prime}}$ only, up to any given order $\mathcal{O}\left(\lambda^{p}\right)$. This helps us to derive the effective symplectic form from Eq. (8)

$$
[\Omega]_{p}=\sum_{a, a^{\prime}}\left[d P_{a i} d \Phi_{a}^{(i)}+d Q_{a^{\prime} i} d \Psi_{a^{\prime}}^{(i)}\right]_{p},
$$

where the bracket $[\cdot]_{p}$ means to replace all higher derivatives of $\Phi_{a}$ and $\Psi_{b}$ by functions of $\Phi_{a}, \dot{\Phi}_{a}$, and $\Psi_{b}$ up to order $\lambda^{p}$. The final Hamiltonian is defined by Eq. (41) with $[\cdot]_{1}$ replaced by $[\cdot]_{p}$. The Hamilton equations will give the equations of motion up to $\mathcal{O}\left(\lambda^{p+1}\right)$.

\section{To all orders: A formal proof}

Now we give a formal proof for the self-consistency of the perturbative formulation. From the equations of motion (20) and (21), assume that one can find an exact solution (to all orders in $\lambda$ )

$$
\ddot{\Phi}_{a}=h_{a}\left(\Phi_{b}, \dot{\Phi}_{b}, \Psi_{b^{\prime}}\right), \quad \dot{\Psi}_{a^{\prime}}=f_{a^{\prime}}\left(\Phi_{b}, \dot{\Phi}_{b}, \Psi_{b^{\prime}}\right)
$$

for certain functions $f$ and $h$ by infinite iteration (or inspiration). From this, higher derivatives of $\Phi_{a}$ and $\Psi_{a^{\prime}}$ can be written as functions on the reduced phase space

$$
\Phi_{a}^{(i)}=h_{a i}\left(\Phi_{b}, \Phi_{b}, \Psi_{b^{\prime}}\right), \quad \Psi_{a^{\prime}}^{(i)}=f_{a^{\prime} i}\left(\Phi_{b}, \Phi_{b}, \Psi_{b^{\prime}}\right) .
$$

The functions $h_{a i}$ and $f_{a^{\prime} i}$ can be obtained recursively 


$$
\begin{aligned}
& h_{a(i+1)}=\left[\frac{d}{d t} h_{a i}\right]=\left(\frac{\partial h_{a i}}{\partial \Phi_{b}} \dot{\Phi}_{b}+\frac{\partial h_{a i}}{\partial \Phi_{b}} h_{b}+\frac{\partial h_{a i}}{\partial \Psi_{b^{\prime}}} f_{b^{\prime}}\right), \\
& f_{a^{\prime}(i+1)}=\left[\frac{d}{d t} f_{a^{\prime} i}\right]=\left(\frac{\partial f_{a^{\prime} i}}{\partial \Phi_{b}} \Phi_{b}+\frac{\partial f_{a^{\prime} i}}{\partial \dot{\Phi}_{b}} h_{b}+\frac{\partial f_{a^{\prime} i}}{\partial \Psi_{b^{\prime}}} f_{b^{\prime}}\right),
\end{aligned}
$$

where we used the Einstein's summation convention and the notation

$$
\left.[A] \equiv A\right|_{\left\{\Phi_{a}^{(i)}=h_{a i}, \Psi_{a^{\prime}}^{(i)}=f_{a^{\prime} i}\right\}} \cdot
$$

A few identities that will come in handy in the proof are the following. From Eqs. (6) and (7) we find

$$
\dot{P}_{a i}=\frac{\partial L_{0}}{\partial \Phi_{a}^{(i)}}-P_{a(i-1)}, \quad \dot{Q}_{a^{\prime} i}=\frac{\partial L_{0}}{\partial \Psi_{a^{\prime}}^{(i)}}-Q_{a^{\prime}(i-1)} .
$$

For an arbitrary function $A$ on the total phase space, we have the following identities:

$$
\begin{gathered}
\frac{d}{d t}[A]=[\dot{A}]+\frac{\partial[A]}{\partial \dot{\Phi}_{a}}\left(\Phi_{a}-h_{a}\right)+\frac{\partial[A]}{\partial \Psi_{a^{\prime}}}\left(\Psi_{a^{\prime}}-f_{a^{\prime}}\right), \\
\frac{\partial[A]}{\partial \Psi_{a^{\prime}}}=\left[\frac{\partial A}{\partial \Phi_{b}^{(i)}}\right] \frac{\partial h_{b i}}{\partial \Psi_{a^{\prime}}}+\left[\frac{\partial A}{\partial \Psi_{b^{\prime}}^{(i)}}\right] \frac{\partial f_{b^{\prime} i}}{\partial \Psi_{a^{\prime}}},
\end{gathered}
$$

where one can also replace $\partial / \partial \Psi_{a^{\prime}}$ by $\partial / \partial \Phi_{a}$ or $\partial / \partial \dot{\Phi}_{a}$ in the last formula. The effective conjugate momenta $p_{0 a}, p_{1 a}$, and $q_{a^{\prime}}$ are defined by

$$
\begin{aligned}
& {\left[\sum_{a, a^{\prime}, i} P_{a i} \delta \Phi_{a}^{(i)}+Q_{a^{\prime} i} \delta \Psi_{a^{\prime}}^{(i)}\right]} \\
& \quad=\sum_{a, a^{\prime}} p_{0 a} \delta \Phi_{a}+p_{1 a} \delta \Phi_{a}+q_{a^{\prime}} \delta \Psi_{a^{\prime}},
\end{aligned}
$$

and they are

$$
\begin{aligned}
p_{0 a} & =\left[P_{b j} \frac{\partial h_{b j}}{\partial \Phi_{a}}+Q_{b^{\prime} j} \frac{\partial f_{b^{\prime} j}}{\partial \Phi_{a}}\right], \\
p_{1 a} & =\left[P_{b j} \frac{\partial h_{b j}}{\partial \dot{\Phi}_{a}}+Q_{b^{\prime} j} \frac{\partial f_{b^{\prime} j}}{\partial \dot{\Phi}_{a}}\right], \\
q_{a^{\prime}} & =\left[P_{b j} \frac{\partial h_{b j}}{\partial \Psi_{a^{\prime}}}+Q_{b^{\prime} j} \frac{\partial f_{b^{\prime} j}}{\partial \Psi_{a^{\prime}}}\right] .
\end{aligned}
$$

The effective Hamiltonian is

$$
H=\left[p_{0 a} \dot{\Phi}_{a}+p_{1 a} \ddot{\Phi}_{a}+q_{a^{\prime}} \dot{\Psi}_{a^{\prime}}-L\right] .
$$

The Hamilton equations based on the symplectic structure (50) are

$$
\begin{aligned}
\left(p_{0 a}\right)^{\cdot}= & -\left(p_{1 b} \frac{\partial h_{b}}{\partial \Phi_{a}}+q_{b^{\prime}} \frac{\partial f_{b^{\prime}}}{\partial \Phi_{a}}\right)+\frac{\partial[L]}{\partial \Phi_{a}} \\
& +\frac{\partial p_{1 b}}{\partial \Phi_{a}}\left(\ddot{\Phi}_{b}-h_{b}\right)+\frac{\partial q_{b^{\prime}}}{\partial \Phi_{a}}\left(\dot{\Psi}_{b^{\prime}}-f_{b^{\prime}}\right), \\
\left(p_{1 a}\right)^{\cdot}= & -\left(p_{1 b} \frac{\partial h_{b}}{\partial \Phi_{a}}+q_{b^{\prime}} \frac{\partial f_{b^{\prime}}}{\partial \Phi_{a}}\right)+\frac{\partial[L]}{\partial \Phi_{a}} \\
& +\frac{\partial p_{1 b}}{\partial \Phi_{a}}\left(\ddot{\Phi}_{b}-h_{b}\right)+\frac{\partial q_{b^{\prime}}}{\partial \Phi_{a}}\left(\dot{\Psi}_{b^{\prime}}-f_{b^{\prime}}\right)-p_{0 a},
\end{aligned}
$$

$$
\begin{aligned}
\left(q_{a^{\prime}}\right)^{\cdot}= & -\left(p_{1 b} \frac{\partial h_{b}}{\partial \Psi_{a^{\prime}}}+q_{b^{\prime}} \frac{\partial f_{b^{\prime}}}{\partial \Psi_{a^{\prime}}}\right)+\frac{\partial[L]}{\partial \Psi_{a^{\prime}}} \\
& +\frac{\partial p_{1 b}}{\partial \Psi_{a^{\prime}}}\left(\ddot{\Phi}_{b}-h_{b}\right)-\frac{\partial q_{b^{\prime}}}{\partial \Psi_{a^{\prime}}}\left(\dot{\Psi}_{b^{\prime}}-f_{b^{\prime}}\right) .
\end{aligned}
$$

With the help of Eqs. (56)-(58), one can show from Eqs. (60), (61), and (62) that Eq. (64) is automatically satisfied, and that Eqs. (65) and (66) are equivalent to the equations of motion (51).

\section{EXAMPLES}

\section{A. A single complex fermion}

Let us consider the case of a single complex fermion in $0+1$ dimension as a simple example. Remarkably, in this case we can describe the effect of arbitrary higher derivative interactions to all orders. Assume that the Lagrangian is of the following form:

$$
L=\frac{i}{2}(\bar{\Psi} \Psi+\Psi \dot{\bar{\Psi}})-m \bar{\Psi} \Psi-\lambda V\left(\bar{\Psi}^{(i)}, \Psi^{(i)}\right),
$$

where $\bar{\Psi}$ is the complex conjugation of $\Psi$. Instead of decomposing the complex fermion into two real fermions, we will maintain the complex structure. By applying integration by parts to the action, we can always rewrite the Lagrangian in such a way that $\Psi \leftrightarrow \Psi$ is a symmetry.

The equations of motion are

$$
i \dot{\Psi}-m \Psi-\lambda \sum_{i=0}^{n}\left(-\frac{d}{d t}\right)^{i} \frac{\partial_{L} V}{\partial \bar{\Psi}^{(i)}}=0
$$

and its complex conjugation. The subscript $L$ of $\partial_{L}$ refers to differentiation from the left.

It is straightforward to see that, by iterating the equations of motion order by order in $\lambda$, the function $f$ defined in Eq. (51) will be of the following form:

$$
\left(\begin{array}{c}
\dot{\Psi} \\
\dot{\bar{\Psi}}
\end{array}\right)=\left(\begin{array}{cc}
R_{1} & R_{2} \\
\bar{R}_{2} & \bar{R}_{1}
\end{array}\right)\left(\begin{array}{c}
\Psi \\
\bar{\Psi}
\end{array}\right)
$$


for some constants $R_{1}, R_{2} \in \mathrm{C}$ due to the anticommutativity of the fermions. This is not the only solution to the exact equations of motion. In general, the equation of motion can be nonlinear, including terms like $\bar{\Psi} \dot{\Psi} \dot{\bar{\Psi}}$, etc., but these terms will not appear in the iteration procedure outlined in previous sections. Analogous to Eq. (52), for higher derivatives we have

$$
\left(\begin{array}{c}
\Psi^{(n)} \\
\Psi^{(n)}
\end{array}\right)=\left(\begin{array}{cc}
R_{1}^{(n)} & R_{2}^{(n)} \\
\bar{R}_{2}^{(n)} & \bar{R}_{1}^{(n)}
\end{array}\right)\left(\begin{array}{c}
\Psi \\
\Psi
\end{array}\right),
$$

where

$$
\left(\begin{array}{cc}
R_{1}^{(n)} & R_{2}^{(n)} \\
\bar{R}_{2}^{(n)} & \bar{R}_{1}^{(n)}
\end{array}\right) \equiv\left(\begin{array}{cc}
R_{1} & R_{2} \\
\bar{R}_{2} & \bar{R}_{1}
\end{array}\right)^{n} .
$$

Note that the effective conjugate momenta (62) must be linear in $\Psi$ and $\bar{\Psi}$ :

$$
\begin{aligned}
& q_{\Psi}=i\left[\lambda b_{2} \Psi+\left(\frac{1}{2}+\lambda b_{1}\right) \bar{\Psi}\right], \\
& q_{\bar{\Psi}}=i\left[\left(\frac{1}{2}+\lambda \bar{b}_{1}\right) \Psi+\lambda \bar{b}_{2} \Psi\right],
\end{aligned}
$$

because there is no nonvanishing cubic term in $\Psi$ and $\bar{\Psi}$. We have imposed the relation $\bar{q}_{\Psi}=-q_{\bar{\Psi}}$ because $q_{\Psi} \delta \Psi$ $+q_{\bar{\Psi}} \delta \bar{\Psi}$ should be Hermitian. The symplectic two-form on the reduced phase space is thus of the form

$$
\begin{aligned}
\Omega= & i\left(\left[1+\lambda\left(b_{1}+\bar{b}_{1}\right)\right] d \bar{\Psi} d \Psi+\lambda b_{2} d \Psi d \Psi\right. \\
& \left.+\lambda \bar{b}_{2} d \bar{\Psi} d \bar{\Psi}\right),
\end{aligned}
$$

for some constants $b_{1}, b_{2} \in \mathbf{C}$.

By a change of variables

$$
\psi=\gamma_{1} \Psi+\gamma_{2} \Psi, \quad \bar{\psi}=\bar{\gamma}_{2} \Psi+\bar{\gamma}_{1} \Psi,
$$

where $\gamma_{1}, \gamma_{2}$ satisfy $^{1}$

$$
\left|\gamma_{1}\right|^{2}+\left|\gamma_{2}\right|^{2}=1+\lambda\left(b_{1}+\bar{b}_{1}\right), \quad \gamma_{1} \bar{\gamma}_{2}=\lambda b_{2},
$$

we have $\Omega=i d \bar{\psi} d \psi$ and so the Poisson bracket is standard

$$
(\bar{\psi}, \psi)=-i,
$$

and others are zeros.

To derive the Hamiltonian, we note that by substituting all derivatives of the fermions according to Eq. (70), the potential becomes

$$
[V]=c \bar{\psi} \psi
$$

${ }^{1} \mathrm{~A}$ solution to Eq. (75) exists if $2\left|\lambda b_{2}\right|<1+\lambda\left(b_{1}+\bar{b}_{1}\right)$. This holds when $\lambda$ is sufficiently small. Otherwise the perturbative approach breaks down. for some real constant $c$ up to a constant. The effective Hamiltonian (63) is also a function of $\psi, \bar{\psi}$, which are the only two variables in the reduced phase space. Hence

$$
H=m^{\prime} \bar{\psi} \psi,
$$

where the effective mass $m^{\prime}$ is given by

$$
\begin{aligned}
m^{\prime}= & \left\{m+\lambda\left[c+i\left(b_{1} R_{1}-\bar{b}_{1} \bar{R}_{1}-b_{2} R_{2}+\bar{b}_{2} \bar{R}_{2}\right)\right]\right\} \\
& \times\left(\left|\gamma_{1}\right|^{2}-\left|\gamma_{2}\right|^{2}\right)^{-1} .
\end{aligned}
$$

The effective Lagrangian is thus

$$
\widetilde{L}=i \bar{\psi} \dot{\psi}-m^{\prime} \bar{\psi} \psi,
$$

which is simplified by integration by parts.

Notice that the change of variables, Eq. (74), similar to a Bogoliubov transformation, results in a change of vacuum upon quantization. If the coefficients of higher derivative terms depend on some background fields, variation of the background fields will induce the creation of particles. The higher derivative terms also contribute to the effective mass $m^{\prime}$.

In the above we see that for two real fermions only the quadratic terms in the potential have some effect in the perturbative approach. In general, if the number of independent fermion degrees of freedom is finite, say $N$, in the perturbative potential we can ignore all interaction terms with more than $N$ factors of fermions since they will all vanish on the reduced phase space. Hence it is possible that a lot of information is lost in the perturbative expansion.

\section{B. Supersymmetric spacetime noncommutative field theory}

Noncommutative field theories [13] have attracted much attention in recent years because of their natural appearance in string theory as the low energy description of D-branes in a $B$ field background [14]. Compared with spatial noncommutativity, field theories with spacetime noncommutativity are much less understood [15], but also particularly interesting in the context of string theory [16]. In terms of the Moyal product, spacetime noncommutativity means infinite time derivatives. In [6], a perturbative approach is applied to the spacetime noncommutative field theory of a scalar field. It would be interesting to consider noncommutative field theories with supersymmetry $[17,18]$ since supersymmetry tends to cure the UV-IR connection problem in the quantum theory [19]. As an example, consider the Wess-Zumino model on $1+1$-dimensional noncommutative spacetime for a real scalar field $\Phi$ and Majorana fermion $\Psi$ with the Lagrangian density

$$
\begin{aligned}
L= & -\frac{1}{2} \partial_{\mu} \Phi * \partial^{\mu} \Phi-\frac{i}{2} \bar{\Psi} * \gamma^{\mu} \partial_{\mu} \Psi-\frac{1}{2}(m \Phi+\lambda \Phi * \Phi) * 2 \\
& -\frac{i}{2} m \bar{\Psi} * \Psi-\left(\frac{i}{2} \lambda \bar{\Psi} * \Psi * \Phi+\text { H.c. }\right)
\end{aligned}
$$

where $\gamma^{\mu}=\left(i \sigma^{2}, \sigma^{1}\right)$ and $\sigma^{i}$ are the Pauli matrices. 
The *-product is defined by

$$
f * g(x)=\left.e^{(i / 2) \theta^{\mu \nu} \partial_{\mu} \partial_{\nu}^{\prime}} f(x) g\left(x^{\prime}\right)\right|_{x^{\prime}=x}
$$

and so

$$
[t, x]_{*}=i \theta .
$$

Up to total derivatives which do not change the action, the Lagrangian (81) can be simplified as

$$
\begin{aligned}
L= & -\frac{1}{2} \partial_{\mu} \Phi \partial^{\mu} \Phi-\frac{i}{2} \bar{\Psi} \gamma^{\mu} \partial_{\mu} \Psi-(m \Phi+\lambda \Phi * \Phi)^{2} \\
& -\frac{i}{2} m \bar{\Psi} \Psi-i \lambda(\bar{\Psi} * \Psi) \Phi .
\end{aligned}
$$

By a field redefinition analogous to Eq. (39), to the lowest order the effective Lagrangian is given by

$$
\begin{aligned}
\widetilde{L} \simeq & -\frac{1}{2} \partial_{\mu} \varphi \partial^{\mu} \varphi-\frac{i}{2} \bar{\psi} \gamma^{\mu} \operatorname{th} \partial_{\mu} \psi-(m \varphi+\lambda \varphi \bar{*} \varphi)^{2} \\
& -\frac{i}{2} m \bar{\psi} \psi-i \lambda(\bar{\psi} \bar{*} \psi) \varphi,
\end{aligned}
$$

where the $\bar{*}$-product is defined as the $*$ in Eq. (82) with the replacement

$$
\begin{aligned}
& \partial_{t}^{n} \varphi \rightarrow \begin{cases}\left(\partial_{x}^{2}-m^{2}\right)^{n / 2} \varphi & (n=\text { even }), \\
\left(\partial_{x}^{2}-m^{2}\right)^{(n-1) / 2} \partial_{t} \varphi & (n=\text { odd }),\end{cases} \\
& \partial_{t}^{n} \psi \rightarrow \begin{cases}\left(\partial_{x}^{2}-m^{2}\right)^{n / 2} \psi & (n=\text { even }), \\
\left(\partial_{x}^{2}-m^{2}\right)^{(n-1) / 2} \gamma^{0}\left(\gamma^{1} \partial_{x}+m\right) \psi \quad(n=\text { odd }),\end{cases}
\end{aligned}
$$

Note that $*$ is related to $\bar{*}$ by the equations of motion at the lowest order. This means that the supersymmery of Eq. (81) guarantees the supersymmetry of Eq. (85) on shell to the first order if we take the new fields $\varphi, \psi$ to transform in the same way as $\Phi, \Psi$.

As we mentioned in Sec. II, the Hamiltonian is always unbounded from below in the canonical formulation, and is thus in contradiction with the usual belief that the Hamiltonian is positive definite as a result of the superalgebra $H$ $\sim Q^{2}$. The only place that could go wrong in the usual argument is the assumption of the supercharge $Q$ to be Hermitian. Indeed, in an example in Sec. II we showed that quantization of the fermions destroys reality conditions. Here we see that the perturbative approach not only provides a consistent quantization but also preserves supersymmetry order by order perturbatively.

\section{String field theory}

As we mentioned in the Introduction, all fields in the interaction term in Witten's bosonic open string field theory [4] are modified by

$$
f \rightarrow \widetilde{f} \equiv e^{a^{2} \partial_{\mu} \partial^{\mu}} f,
$$

where $a^{2}=\ln (3 \sqrt{3} / 4) \alpha^{\prime}$. Similarly, in the bosonic closed string field theory [20], the same form (86) appears with $a^{2}$ $=\frac{1}{2} \ln (3 \sqrt{3} / 4) \alpha^{\prime}[21]$.

The exponent $\partial \cdot \partial \simeq E^{2}-p^{2}$ implies that, after Wick rotation, contribution from the UV excitations of string theory is suppressed. It helps string theory to avoid the UV divergences present in most ordinary field theories. Another way to look at the effect of Eq. (86) is to view $\widetilde{f}$ as the fundamental field variable, and its propagator is modified,

$$
\frac{1}{p_{E}^{2}+m^{2}} \rightarrow \frac{1}{p_{E}^{2}+m^{2}} e^{-2 a^{2} p_{E}^{2}}
$$

which also implies a suppression of high energy modes. However, the appearance of the higher derivative terms also means that we are not sure how to treat this theory exactly [7].

For Witten's bosonic open string field theory, the technique of level truncation was shown to be very effective in the calculation of tachyon potential $[5,22]$. In this calculation only the zero mode of each field has to be considered. A possible, although only weakly supportive reason why the level truncation technique is effective is that the coupling constants are suppressed by a factor of $4 / 3 \sqrt{3} \simeq 0.77$ when we increase the level number [5].

According to the perturbative approach, to the lowest order, we simply replace the factor $\partial_{\mu} \partial^{\mu}$ in Eq. (86) by the mass squared of the field $f$. For instance, the level $(0,0)$ truncation gives the tachyon potential

$$
V=-\frac{1}{2 \alpha^{\prime}} \Phi^{2}+\frac{1}{3}\left(\frac{3 \sqrt{3}}{4}\right)^{3} \widetilde{\Phi}^{3} \rightarrow \frac{1}{2 \alpha^{\prime}} \varphi^{2}+\frac{1}{3} \varphi^{3},
$$

where we have used our results of first order approximation in the perturbative approach to replace $\Phi$ by $\varphi$ according to Eq. (39) and $\partial \cdot \partial$ by $m^{2}=-1 / \alpha^{\prime}$ for the tachyon field $\Phi$. Note that this replacement is not suitable for the zero mode of $\Phi$ because the zero mode is determined solely by the potential term, and is thus a nonperturbative effect. In principle, the zero mode should be treated first before the perturbative approach is applied. Here we are considering fluctuations around the false vacuum.

For a field with $m^{2}=n / \alpha^{\prime}$, from Eq. (86) we have

$$
e^{a^{2} \partial \partial^{\mu}} \rightarrow\left(\frac{3 \sqrt{3}}{4}\right)^{n}
$$

This implies that the interaction for higher excitation modes are strengthened by factors of $3 \sqrt{3} / 4$ when we increase the level number. Therefore, combined with the decrease in coupling, fluctuation modes other than the zero modes will have roughly the same effective interaction when we increase the level number. If the reason why level truncation works for tachyon potential is really the one mentioned above, we will not expect level truncation to be effective for fluctuation modes. 


\section{EXTENSION OF THE PERTURBATIVE APPROACH}

In this section we generalize our formal proof of all orders in Sec. III C to the situation where the reduced phase space is allowed to keep derivatives of fields up to an arbitrary given order. For each field (boson or fermion) $q_{a}$ we specify an integer $K_{a}>1$ so that $q_{a}, \dot{q}_{a}, \ldots, q_{a}^{\left(K_{a}-1\right)}$ are kept in the reduced phase space. Using the equations of motion, in principle we can rewrite the $K_{a}$ th derivative of $q_{a}$ as a function on the reduced phase space

$$
q_{a}^{\left(K_{a}\right)}=f_{a}\left(q_{b}^{(j)}\right)
$$

where $j$ is less than $K_{b}$, analogous to Eq. (51).

Higher derivatives of $q_{a}$ can be derived from Eq. (90),

$$
q_{a}^{(i)}=f_{a i}\left(q_{b}^{(j)}\right)
$$

and the functions $f_{a i}$ can be obtained recursively,

$$
\begin{aligned}
f_{a(i+1)} & =\left[\frac{d}{d t} f_{a i}\right] \\
& =\sum_{b} \sum_{j=0}^{k_{b}-2} \frac{\partial f_{a i}}{\partial q_{b}^{(j)}} q_{b}^{(j+1)}+\sum_{b} \frac{\partial f_{a i}}{\partial q_{b}^{\left(K_{b}-1\right)}} f_{b},
\end{aligned}
$$

where we used the notation

$$
\left.[A] \equiv A\right|_{q_{a}^{(i)}=f_{a i}} .
$$

From Eqs. (22) and (23), we find

$$
\dot{p}_{a i}=\frac{\partial L_{0}}{\partial q_{a}^{(i)}}-p_{a(i-1)},
$$

where $p_{a i}$ is the $i$ th canonical momentum of $q_{a}$.

For an arbitrary function $A$ on the phase space, we find the following identities:

$$
\begin{gathered}
\frac{d}{d t}[A]=[\dot{A}]+\sum_{a} \frac{\partial[A]}{\partial q_{a}^{\left(K_{a}-1\right)}}\left(q_{a}^{\left(K_{a}\right)}-f_{a}\right), \\
\frac{\partial[A]}{\partial q_{a}^{(i)}}=\sum_{b=1} \sum_{j=0}\left[\frac{\partial A}{\partial q_{b}^{(j)}}\right] \frac{\partial f_{b j}}{\partial q_{a}^{(i)}} .
\end{gathered}
$$

The perturbative momentum $\Pi_{a i}$ can be read off from

$$
\sum_{b, j} p_{b j} \delta q_{b}^{(j)}=\sum_{a} \sum_{i=0}^{K_{a}-1} \Pi_{a i} \delta q_{a}^{(i)},
$$

and we find

$$
\Pi_{a i}=\sum_{b, j}\left[p_{b j} \frac{\partial q_{b}^{(j)}}{\partial q_{a}^{(i)}}\right]
$$

The Hamiltonian is

$$
H=\left[\sum_{a} \sum_{i=0}^{K_{a}-1} \Pi_{a i} q_{a}^{(i+1)}-L\right] .
$$

The symplectic structure is

$$
\begin{aligned}
\Omega= & \sum_{b, j} d p_{b j} d q_{b}^{(j)} \\
= & \sum_{a} \sum_{i=0}^{K_{a}-1} d \Pi_{a i} d q_{a}^{(i)} \\
= & \sum_{a} \sum_{i=0}^{K_{a}-1} \sum_{b} \sum_{j=0}^{K_{b}-1} d q_{a}^{(i)} \frac{(-1)^{a}}{2} \\
& \times\left(\sum^{(-1)^{a} \cdot b} \frac{\partial \Pi_{b j}}{\partial q_{a}^{(i)}}-\frac{\partial \Pi_{a i}}{\partial q_{b}^{(j)}}\right) d q_{b}^{(j)} \\
= & \sum_{a} \sum_{i=0}^{K_{a}-1} \sum_{b} \sum_{j=0}^{K_{b}-1} d q_{a}^{(i)} \Omega_{a_{i} b_{j}} d q_{b}^{(j)},
\end{aligned}
$$

where

$$
\Omega_{a_{i} b_{j}}=\frac{(-1)^{a}}{2}\left((-1)^{a \cdot b} \frac{\partial \Pi_{b j}}{\partial q_{a}^{(i)}}-\frac{\partial \Pi_{a i}}{\partial q_{b}^{(j)}}\right) .
$$

In the above, when $a, b$ appear as the power of $(-1)$, they are identified with 0 or 1 depending on whether $q_{a}$ is a boson or fermion.

The Hamilton equations based on the symplectic structure (101) are

$$
\begin{aligned}
\left(\Pi_{a i}\right) \cdot= & \sum_{b}\left\{-\left(\Pi_{b\left(K_{b}-1\right)} \frac{\partial f_{b}}{\partial q_{a}^{(i)}}\right)\right. \\
& \left.+(-1)^{(a \cdot b)} \frac{\Pi_{b\left(K_{b}-1\right)}}{\partial q_{a}^{(i)}}\left(q_{b}^{\left(K_{b}\right)}-f_{b}\right)\right\} \\
& +\frac{\partial[L]}{\partial q_{a}^{(i)}}-\Pi_{a(i-1)} .
\end{aligned}
$$

With the help of Eqs. (94)-(96), one can show from Eq. (98) that Eq. (102) is equivalent to the relation (90) for $i \neq 0$, and the case $i=0$ gives only an identity.

\section{ACKNOWLEDGMENTS}

The authors thank Koji Hashimoto, Marc Henneaux, and John Wang for helpful discussions. This work was supported in part by the National Science Council, the CosPA project of the Ministry of Education, the National Center for Theoretical Sciences, Taiwan, Republic of China, and the Center for Theoretical Physics at National Taiwan University. 
[1] M. Ostrogradski, Mem. Ac. St. Petersbourg, VI 4, 385 (1850).

[2] C.S. Chu, J. Lukierski, and W.J. Zakrzewski, Nucl. Phys. B632, 219 (2002).

[3] A.D. Fokker, Z. Phys. 58, 386 (1929); A. Pais and G.E. Uhlenbeck, Phys. Rev. 79, 145 (1950); K.S. Stelle, Phys. Rev. D 16, 953 (1977); E.S. Fradkin and A.A. Tseytlin, Nucl. Phys. B201, 469 (1982); J. Julve and M. Tonin, Nuovo Cimento Soc. Ital. Fis., B 46B, 137 (1978).

[4] E. Witten, Nucl. Phys. B268, 253 (1986).

[5] V.A. Kostelecky and S. Samuel, Nucl. Phys. B336, 263 (1990).

[6] T.C. Cheng, P.M. Ho, and M.C. Yeh, Nucl. Phys. B625, 151 (2002).

[7] D.A. Eliezer and R.P. Woodard, Nucl. Phys. B325, 389 (1989).

[8] C.N. Yang and D. Feldman, Phys. Rev. 79, 972 (1950).

[9] D. Bahns, S. Doplicher, K. Fredenhagen, and G. Piacitelli, Phys. Lett. B 533, 178 (2002).

[10] C.h. Rim and J.H. Yee, hep-th/0205193.

[11] J. Wess and B. Zumino, Phys. Lett. 49B, 52 (1974).

[12] L.D. Faddeev and R. Jackiw, Phys. Rev. Lett. 60, 1692 (1988); M. Henneaux and C. Teitelboim, Quantization Of Gauge Systems (Princeton University, Princeton, NJ, 1992).

[13] For a review of noncommutative field theories, see: M.R. Douglas and N.A. Nekrasov, Rev. Mod. Phys. 73, 977 (2002).

[14] A. Connes, M.R. Douglas, and A. Schwarz, J. High Energy Phys. 02, 003 (1998); N. Seiberg and E. Witten, ibid. 09, 032 (1999).

[15] J.L. Barbon and E. Rabinovici, Phys. Lett. B 486, 202 (2000);
J. Gomis and T. Mehen, Nucl. Phys. B591, 265 (2000); M. Chaichian, A. Demichev, P. Presnajder, and A. Tureanu, Eur. Phys. J. C 20, 767 (2001); L. Alvarez-Gaume, J.L. Barbon, and R. Zwicky, J. High Energy Phys. 05, 057 (2001); T. Mateos and A. Moreno, Phys. Rev. D 64, 047703 (2001); A. Bassetto, L. Griguolo, G. Nardelli, and F. Vian, J. High Energy Phys. 07, 008 (2001); M. Li, ibid. 05, 033 (2002).

[16] N. Seiberg, L. Susskind, and N. Toumbas, J. High Energy Phys. 06, 044 (2000); N. Seiberg, L. Susskind, and N. Toumbas, ibid. 06, 021 (2000); R. Gopakumar, J. Maldacena, S. Minwalla, and A. Strominger, ibid. 06, 036 (2000); R. Gopakumar, S. Minwalla, N. Seiberg, and A. Strominger, ibid. 08, 008 (2000).

[17] I. Chepelev and R. Roiban, J. High Energy Phys. 05, 037 (2000).

[18] C.S. Chu and F. Zamora, J. High Energy Phys. 02, 022 (2000).

[19] H.O. Girotti, M. Gomes, V.O. Rivelles, and A.J. da Silva, Nucl. Phys. B587, 299 (2000); J. High Energy Phys. 05, 040 (2002).

[20] T. Kugo and K. Suehiro, Nucl. Phys. B337, 434 (1990); T. Kugo, H. Kunimoto, and K. Suehiro, Phys. Lett. B 226, 48 (1989); M. Saadi and B. Zweibach, Ann. Phys. (N.Y.) 192, 213 (1989); M. Kaku, in Functional Integration, Geometry and Strings, edited by Z. Haba and J. Sobczyk (Birkhauser, Boston, 1989); M. Kaku, Phys. Rev. D 41, 3734 (1990).

[21] V.A. Kostelecky and S. Samuel, Phys. Rev. D 42, 1289 (1990).

[22] L. Rastelli, A. Sen, and B. Zwiebach, hep-th/0102112. 